Bundesgesundheitsbl -

Gesundheitsforsch - Gesundheitsschutz

2001 • 44:813- 822 ๑ Springer-Verlag 2001
Originalien und Ubersichtsarbeiten

B.-M. Kurth · Robert Koch-Institut, Berlin

\section{Demographischer Wandel und Anforderungen an das Gesundheitswesen}

\section{Zusammenfassung}

Das Statistische Bundesamt prognostizierte eine verlängerte individuelle Lebenserwartung der Bevölkerung der Bundesrepublik Deutschland, einhergehend mit einer Verringerung des Anteilsjüngerer Menschen. Das wirft die Frage auf, ob diese Entwicklung zu einer verstärkten Inanspruchnahme des Gesundheitswesens führt, die möglicherweise künftig nicht mehr finanzierbar sein wird. Es wird in dieser Stellungnahme versucht, ausschließlich aufgrund vorliegender Gesundheitsdaten eine Antwort zu geben. Dabei wird festgestellt, dass ein längeres Leben nicht unbedingt einhergeht mit einem verlängerten Abschnitt in Krankheit und Pflegebedürftigkeit. Es gibt vielmehr Anhaltspunkte dafür, dass der Alterungsprozess der Bevölkerung nicht zu einem proportionalen Anstieg der Krankheits- und Behinderungslast für das gesundheitlicheVersorgungssystem führen muss. Dennoch wird die Erhöhung des Anteils Älterer an der Gesamtbevölkerung, der nicht nur durch die wachsende Lebenserwartung als Ausdruck kultureller, medizinischer und sozialer Entwicklung verursacht ist, sondern auch durch den Rückgang der Geburtenraten, alsbald Anforderungen an die Finanzierung des Gesundheitswesens stellen, über deren Größenordnung es wenig belastbare Daten gibt.

\section{Schlüsselwörter}

Demographischer Wandel · Morbidität . Mortalität · Lebensqualität · Inanspruchnahme des Gesundheitswesens ie vom Statistischen Bundesamt vorgelegten Schätzungen für die demographische Entwicklung der Bundesrepublik Deutschland bis zum Jahr 2050 [1] prognostizieren zum einen eine weitere Lebensverlängerung der Einzelindividuen, zum anderen eine Verringerung des Anteils Jüngerer und damit der Bevölkerungszahl überhaupt wegen zu niedriger Geburtenzahlen. Diese beiden Aspekte wirken sich zwar komplex auf die zu erwartenden Anforderungen an das Gesundheitssystem aus, sind vorerst aber voneinander $\mathrm{zu}$ trennen. Der Lebensverlängerungsprozess ist der allgemeinste demographische Ausdruck der sozialen Entwicklung in menschlichen Kulturen, und steigende Lebenserwartungswerte sind stets mit Fortschritten menschlicher Kulturen gleichzusetzen. Im Wettstreit der einzelnen Kulturkreise im gegenwärtigen Prozess einer forcierten Globalisierung ist das erreichte Niveau der Lebenserwartung bzw. der Sterblichkeit das bestimmende und objektive gesellschaftliche Gütekriterium. Damit ist die steigende Lebenserwartung in der Bundesrepublik Deutschland ein wertvolles Gut, das kontinuierlich zu sichern ist. $\mathrm{Zu}$ hinterfragen ist, inwieweit dieser Lebensverlängerungsprozess mit einem Zugewinn an "gesunden“ Lebensjahren verbunden ist, mit welcher Lebensqualität die zusätzlich gewonnenen Lebensjahre verbracht werden können.

\section{Lebensverlängerung und Gesundheitszustand}

Die Antwort auf die naheliegende Frage, $o b$ ein längeres Leben einen längeren $\mathrm{Ab}$ schnitt von Jahren in Krankheit und Pflegebedürftigkeit bedeutet, ist keine Glaubensfrage und auch keine Frage des unterschiedlichen Theorienzugangs, sondern abhängig von den vorliegenden Informationen. Die in der Epidemiologie als Kohortenstudie benannte Art der Beobachtung von Lebensverläufen verschiedener Geburtsjahrgänge wäre eine ideale Datengrundlage, denn sie kann Informationen liefern über die durchschnittliche Verhaltens-, Morbiditäts- und Lebensqualitätsentwicklung von Probanden mit einer langen Lebenszeit im Vergleich zu denen mit einem vorzeitigen oder zumindest früher liegenden Tod. Daraus lieBen sich fundierte qualitative und quantitative Prognosen über künftig zu erwartende Lebensverläufe ableiten und die Frage nach dem Zusammenhang zwischen Lebensverlängerung und individueller Gesundheit leichter beantworten. Eine solche Kohortenerhebung in großem Umfang ist sehr kostenaufwendig und existiert daher in Deutschland bislang nicht. Es muss also auf andere Datenquel-

\footnotetext{
Dr. Bärbel-Maria Kurth

Abteilung für Epidemiologie

und Gesundheitsberichterstattung,

Robert Koch-Institut, Postfach 6502 80,

13302 Berlin, E-Mail: Kurthb@rki.de
} 
Bundesgesundheitsbl -

Gesundheitsforsch - Gesundheitsschutz

2001 · 44:813- $822 \odot$ Springer-Verlag 2001

\section{B.-M. Kurth}

Demographic changes

and their consequences for the german health care system

\section{Abstract}

The Federal Statistical Office is forecasting an increase of individual life expectancy for Germans, paralleled by a decrease of the proportion of young people in the population. The question arises, whether this development will increase the strain on the German Health system in a way that cannot be financed in the future. This memorandum tries to answer this question solely on the basis of existing health data. It is stated that an extended life span is not necessarily associated with a longer time period of illness and disability. There are indications that the increasing ageing of the population does not lead to a proportional rise in expenditure in health care for the elderly. The increase of the proportion of elderly people does not only reflect improved life expectancy as a consequence of cultural, medical and social developments. Instead, reduction of birth rates will significantly impose constraints of the Health System, the extend of which cannot at present be estimated due to the scarcity of evidence based knowledge.

\section{Keywords}

Demographic Changes · Morbidity · Mortality · Quality of Life · Consumption of Health Care Service

Originalien und Übersichtsarbeiten

\section{Multimorbidität* nach Alter und Geschlecht}

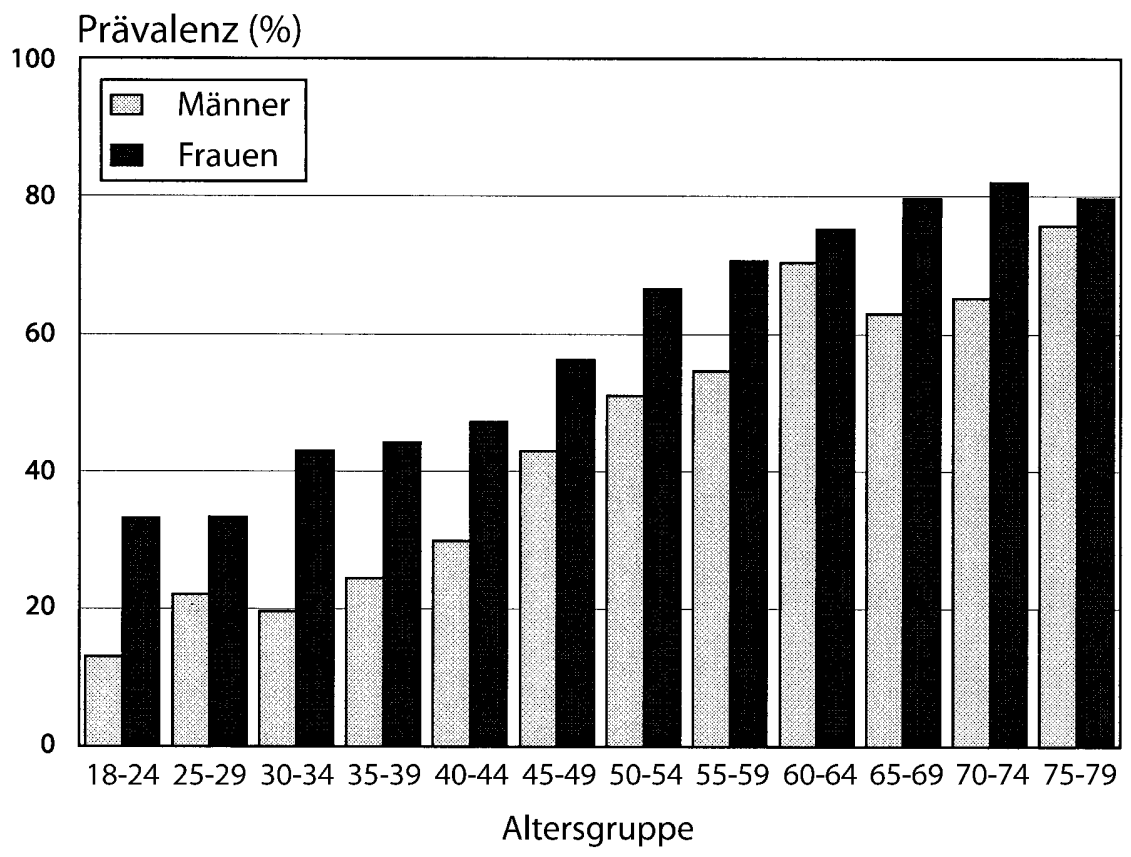

*Multimorbidität: Mindestens 2 Krankheiten des Spektrums (45 Krankheiten) traten in den letzten 12 Monaten auf

Abb.1 $\Delta$ Multimorbidität $^{1}$ nach Alter und Geschlecht

len zurückgegriffen werden, um zumindest Anhaltspunkte für den veränderten Gesundheitszustand in Abhängigkeit vom Lebensalter zu geben.

\section{Strukturelle Veränderungen in der Mortalität}

Eine mögliche Informationsquelle ist die Todesursachenstatistik des Statistischen Bundesamtes, aus der man bereits über einen Verlauf von nur sechs Jahren einen strukturellen Wandel in den Anteilen der Todesursachengruppen in der Alterssterblichkeit (hier definiert als Tod nach Vollendung des 65. Lebensjahres) feststellen kann. Aus Tabelle 1 ist ersichtlich, dass die Gruppe der Erkrankungen des Kreislaufsystems zwar nach wie vor absolut die häufigste Todesursache darstellt, dass sich ihr Anteil am Gesamtspektrum aller Todesursachen aber rasant verringert. Der Rückgang des Anteils der Erkrankungen des Kreislaufsystems an der vorzeitigen Sterblichkeit (Tod vor Vollendung des 65. Lebensjah-

\footnotetext{
1 Multimorbidität: Mindestens 2 Krankheiten des Spektrums (45 Krankheiten) traten in den letzten 12 Monaten auf.
}

res) ist mit Ursache für die verlängerte Lebenserwartung. Auch bei der Alterssterblichkeit nimmt die Bedeutung der Krankheiten des Kreislaufsystems ab. Dafür nehmen aber Krankheiten des Nervensystems und psychische Krankheiten als Ursachen bei der Alterssterblichkeit ein höheres Gewicht ein.

\section{„Der Anteil der Erkrankungen des Kreis- laufsystems am Gesamtspektrum al- ler Todesursachen verringert sich, die Bedeutung der Krankheiten des Ner- vensystems und psychischer Krankhei- ten nimmt zu."}

Die in Tabelle 1 dargestellten veränderten Anteile der verschiedenen Haupttodesursachen an der standardisierten Sterblichkeit reflektieren deren absolute Bedeutung bei weitem noch nicht im vollen Ausmaß. Nimmt man das Beispiel Krebs (Neubildungen), so ist in Tabelle 1 eine prozentuale Verringerung der altersstandardisierten Sterblichkeit sowohl im jüngeren Lebensalter (dort sehr viel stärker) als auch im höheren Lebensalter zu verzeichnen. Berücksichtigt man aber die sich verändernde demographische Struktur der Bevölkerung 
und damit den absolut wachsenden Teil von Personen im höheren Alter, so verdeutlicht Tabelle 2, dass beispielsweise trotz prozentualer Abnahme des Anteils der Todesursache Krebs an der standardisierten Alterssterblichkeit für Männer die absolute Zahl der Krebssterbefälle gestiegen ist und weiter steigen wird.

Insgesamt lässt sich über die sich zeitlich verändernde Todesursachenstatistik ein verändertes Morbiditätsgeschehen vor dem Tod und damit auch ein verändertes Anforderungsprofil an das Gesundheitssystem ableiten. Der allgemeine soziale und medizinische Fortschritt und auch die gestiegene individuelle Sensibilität in der Bevölkerung bezüglich gesundheitlicher Belange haben den Gesundheitszustand in den gewonnenen Lebensphasen soweit verbessert, dass die lebensbedrohlichen bzw. die mit der Lebensfortführung nicht zu vereinbarenden Krankheitsereignisse zunehmend auf einen Zeitpunkt kurz vor Eintritt des Todes verschoben werden. Dieses modifizierte „Compressed-morbidity-Modell“ ist für die Bundesrepublik Deutschland und vergleichbare Länder charakteristisch. Der Aussagehorizont der Kompressionstheorie erstreckt sich aber nur auf lebensbegrenzende Krankheiten/Krankheitsepisoden. Inwieweit die Jahre davor durch Multimorbidität oder krankheitsfreie Episoden gekennzeichnet sind, ist aus der Mortalitätsstatistik nicht abzuleiten.

\section{Multimorbidität im höheren Alter}

Die Chance für das Auftreten von Langzeitkrankheiten kann infolge niedriger Mortalität bzw. besserer Lebenserwartungswerte höher sein. Längere Überlebenszeiten bei chronischen Leiden bewirken höhere Prävalenzraten an multimorbiden Zuständen. Die Multimorbidität kann als Indikator für die Gesamtmorbidität der Bevölkerung gelten. Es gibt derzeit nur wenige $\mathrm{Da}$ tenquellen, die Aussagen über das Auftreten von Multimorbidität in der Bevölkerung zulassen, eine davon ist die 1998 vom Robert Koch-Institut durchgeführte repräsentative Erhebung zum Gesundheitszustand der erwachsenen Wohnbevölkerung im Alter zwischen 18 und 80 Jahren $[2,3]$. Dabei lässt sich in definierten Lebensphasen eine Zunah- me bzw. Abnahme der Prävalenz der multimorbiden Population in der Bundesrepublik Deutschland feststellen. Bei der männlichen Bevölkerung zeigt sich eine deutliche Zunahme der altersspezifischen Prävalenzraten bei den Übergängen der Altersgruppen von 18 bis $24 \mathrm{zu} 25$ bis 30 Jahren, 40 bis $44 \mathrm{zu}$ 45 bis 49 Jahren, 45 bis 49 zu 50 bis 54 Jahren, 55 bis $59 \mathrm{zu} 60$ bis 64 Jahren und 70 bis $74 \mathrm{zu} 75$ bis 79 Jahren. Bei der weiblichen Bevölkerung sind nur drei Altersübergänge mit besonders markanten Erhöhungen der Multimorbidität verbunden: 25 bis $29 \mathrm{zu} 30$ bis 34 Jahren, 40 bis $44 \mathrm{zu} 45$ bis 49 Jahren und 45 bis $49 \mathrm{zu} 50$ bis 54 Jahren. Auffällig ist die starke Zunahme der Multimorbidität im Altersbereich 40 bis 55 Jahre bei Männern und Frauen (siehe dazu Abb. 1).

Danach ist die Multimorbidität, d.h. das Nebeneinanderbestehen mehrerer chronischer Krankheiten eher die Regel als die Ausnahme. Die durchschnittliche Anzahl von Krankheiten liegt bei den 20- bis 30-Jährigen bei 1,04, bei den 70bis 80-Jährigen bei 3,4 (Tabelle 3). Aus dieser Tabelle, die nur eine Auswahl der

Tabelle 1

Gesamtsterblichkeit (alle Altersklassen), vorzeitige Sterblichkeit (1 bis <65 Jahre) und Alterssterblichkeit ( $\geq 65$ Jahre) an natürlichen krankheitsbedingten Todesursachen je 100000 der Bevölkerung der Bundesrepublik Deutschland 1997 und Veränderung gegenüber 1991

\begin{tabular}{|c|c|c|c|c|c|c|}
\hline \multirow[t]{2}{*}{ Krankheitsklasse } & \multicolumn{3}{|c|}{ Art und zeitlicher Typ der Sterblichkeit ${ }^{1)} 1997$} & \multicolumn{3}{|c|}{ Prozentuale Veränderung 1997 zu 1991} \\
\hline & $\begin{array}{l}\text { Gesamt- } \\
\text { sterblichkeit }\end{array}$ & $\begin{array}{l}\text { Vorzeitige } \\
\text { Sterblichkeit }\end{array}$ & $\begin{array}{l}\text { Alters- } \\
\text { sterblichkeit }\end{array}$ & $\begin{array}{l}\text { Gesamt- } \\
\text { sterblichkeit }\end{array}$ & $\begin{array}{l}\text { Vorzeitige } \\
\text { Sterblichkeit }\end{array}$ & $\begin{array}{l}\text { Alters- } \\
\text { sterblichkeit }\end{array}$ \\
\hline \multicolumn{7}{|c|}{ Neubildungen (ICD-9: 140-208) } \\
\hline Männer & 251,69 & 95,71 & $1.527,05$ & $-7,57$ & $-10,85$ & $-5,85$ \\
\hline Frauen & 150,61 & 68,09 & 827,76 & $-7,92$ & $-9,09$ & $-7,15$ \\
\hline \multicolumn{7}{|c|}{ Endokrine Ernährungs- und Stoffwechselkrankheiten (ICD-9: 240-279) } \\
\hline Männer & 21,60 & 6,39 & 144,59 & $+14,77$ & $+5,10$ & $+18,81$ \\
\hline Frauen & 19,24 & 3,60 & 145,37 & $+0,37$ & $-7,93$ & $+2,22$ \\
\hline \multicolumn{7}{|c|}{ Psychische Krankheiten (ICD-9: 290-319) } \\
\hline Männer & 16,57 & 12,71 & 49,68 & $+0,55$ & $-8,63$ & $+26,54$ \\
\hline Frauen & 6,45 & 3,26 & 32,78 & $+10,82$ & $-6,32$ & $+29,92$ \\
\hline \multicolumn{7}{|c|}{ Krankheiten des Nervensystems und der Sinnesorgane (ICD-9: 320-389) } \\
\hline Männer & 17,21 & 5,32 & 112,97 & $+12,19$ & $-11,04$ & $+25,62$ \\
\hline Frauen & 11,02 & 3,51 & 71,73 & $+4,85$ & $-14,81$ & $+16,50$ \\
\hline \multicolumn{7}{|c|}{ Krankheiten des Kreislaufsystems (ICD-9: 390-459) } \\
\hline Männer & 412,29 & 88,55 & 3043,82 & $-17,55$ & $-22,03$ & $-16,43$ \\
\hline Frauen & 262,82 & 30,68 & 2144,99 & $-16,96$ & $-22,13$ & $-16,33$ \\
\hline
\end{tabular}

1) standardisiert auf die „alte Europastandardbevölkerung“". 
Tabelle 2

Zahl der Sterbefälle und standardisierte Sterbeziffern ${ }^{1)}$ an bösartigen Neubildungen ${ }^{2)}$ und Krankheiten des Kreislaufsystems ${ }^{3)}$, Bundesrepublik Deutschland, 1991- 1998

\begin{tabular}{|c|c|c|c|c|}
\hline & \multicolumn{2}{|c|}{ Bösartige Neubildungen } & \multicolumn{2}{|c|}{ Krankheiten des Kreislaufsystems } \\
\hline & $\begin{array}{l}\text { Zahl der } \\
\text { Sterbefälle }\end{array}$ & $\begin{array}{l}\text { Standardisierte } \\
\text { Sterbeziffer } \\
\text { pro } 100000\end{array}$ & $\begin{array}{l}\text { Zahl der } \\
\text { Sterbefälle }\end{array}$ & $\begin{array}{l}\text { Standardisierte } \\
\text { Sterbeziffer } \\
\text { pro } 100000\end{array}$ \\
\hline \multicolumn{5}{|l|}{ Männer } \\
\hline 1991 & 105212 & 272,30 & 190384 & 500,10 \\
\hline 1998 & 108830 & 249,90 & 169301 & 401,40 \\
\hline Differenz absolut & +3618 & $-22,40$ & -21083 & $-98,70$ \\
\hline Veränderung in \% & $+3,44$ & $-8,23$ & $-11,070$ & $-19,74$ \\
\hline \multicolumn{5}{|l|}{ Frauen } \\
\hline 1991 & 105325 & 163,60 & 265390 & 316,50 \\
\hline 1998 & 103918 & 150,60 & 242103 & 258,10 \\
\hline Differenz absolut & -1407 & $-13,00$ & -23.287 & $-58,40$ \\
\hline Veränderung in \% & $-1,340$ & $-3,02$ & $-8,770$ & $-18,45$ \\
\hline
\end{tabular}

erfragten Krankheitsbilder zeigt, wird ersichtlich, dass der stärkste Alterseinfluss bei den kardiovaskulären Krankheiten vorliegt, in denen eine deutliche Zunahme zu verzeichnen ist. Dementsprechend vergrößert sich auch in den älteren Altersgruppen die Inanspruchnahme entsprechender medizinischer Leistungen.

\section{„Ob die Lebenszeitverlängerung den Zeitpunkt des Einsetzens chronischer Krankheiten nach hinten verschiebt, oder ob die Lebenszeit mit diesen Krankheiten länger wird, bleibt offen."}

Offen bleibt an dieser Stelle die Frage, ob sich im Zuge der prognostizierten Lebenszeitverlängerung der Zeitpunkt des Einsetzens dieser Krankheiten nach hinten verschiebt oder ob die Lebenszeit mit diesen Krankheiten einfach länger wird. Zur Beantwortung dieser Frage bedarf es zumindest einer wiederholten (besser noch einer mehrfachen) Querschnittserhebung, um auf Grundlage eines evtl. vorhandenen Trends künftige Entwicklungen prognostizieren zu können. Die Datenlage hierzu ist relativ spärlich.
Sowohl im Gesundheitssurvey 1991/92 als auch im Bundes-Gesundheitssurvey 1998 wurden verschiedene Krankheiten erfragt. Dabei wuchs über einen relativ kurzen Zeitraum von acht Jahren der Anteil derjenigen Personen im Alter zwischen 65 und 70 Jahren ohne Krankheit, während der hohe Prozentsatz multimorbider Personen leicht zurückging (Tabelle 4).

Dies geht konform mit dem Ansatz von Dinkel, der die über einen Zeitraum von 18 Jahren verfügbaren Daten des Mikrozensus benutzt [4] um festzustellen, ob die längere Lebenszeit verbunden ist mit einer besseren Gesundheit. Auf die im Rahmen des Mikrozensus sehr allgemein gestellte Frage „Sind Sie im Moment krank oder unfallverletzt?" antworten später geborene Jahrgänge gleichen Alters weniger häufig mit „ja“ als ihre jeweiligen Vorgänger. Dies ist nach Dinkels Interpretation zumindest ein Anhaltspunkt dafür, dass der Gesundheitszustand einen Kohorteneffekt in dem Sinne aufweist, dass spätere Geburtsjahrgänge (d. h. die künftigen „Alten") sich länger guter Gesundheit erfreuen als früher Geborene.

Optimistisch stimmt zudem die Tatsache, dass die 65- bis 70-Jährigen, die 1998 nach ihrer Zufriedenheit mit der eigenen Gesundheit befragt wurden, zu ei- nem größeren Anteil zufrieden bzw. sehr zufrieden waren als ihre Altersgenossen noch 1990/92 (Tabelle 5).

Zudem gibt der Zusammenhang mit der Lebenszufriedenheit insgesamt (Tabelle 6) den Hinweis darauf, dass positive Lebensumstände ältere Menschen vermehrt und ansteigend "gesünder" verbleiben lassen. Angemerkt werden muss an dieser Stelle, dass am Gesundheitssurvey nur Personen teilnehmen konnten, die zu Hause in einer eigenen Wohnung lebten. Damit fallen ältere Menschen in Krankenhäusern, geriatrischen Einrichtungen und Pflegestationen aus unserer Erhebung heraus.

\section{Demographischer Wandel und Anforderungen an das Gesundheitssystem}

Auch wenn die Kompressionstheorie für die Bundesrepublik Deutschland Bestätigung finden sollte und unter der optimistischen Annahme, dass höheres Alter in der Zukunft mit einem durchschnittlich besseren Gesundheitszustand einhergeht als heute in der gleichen Altersgruppe, stellt allein die wachsende absolute Anzahl von Hochbetagten in der künftigen Gesellschaft höhere Anforderungen an das Gesundheitswesen. Dies soll am Beispiel der zu erwartenden Krebserkrankungen belegt werden.

Die vom Statistischen Bundesamt in der aktualisierten 9. Bevölkerungsvorausberechnung [1] zur Verfügung gestellten Schätzungen für die demographische Entwicklung der Bundesrepublik bis 2050 stellen die Grundlage für nachfolgende Modellrechnungen dar. Diese Berechnungen basieren auf Annahmen, deren Überprüfung ebenfalls erst in der Zukunft möglich sein wird. Da die Enquete-Kommission „Demographischer Wandel“ in ihrem zweiten Zwischenbericht [5] selbst auf die Problematik der Treffsicherheit von Prognosen hingewiesen hat, sei an dieser Stelle nur angemerkt, dass die Ergebnisse lediglich qualitativ auf die Dimension zu erwartender Entwicklungen hinweisen sollen und nicht als quantitativ belastbare Prognosen zu werten sind. Der Prognosezeitraum ist ebenfalls aus diesem Grunde auf 20 Jahre verkürzt worden. 
Die Entwicklung der Krebsneuerkrankungen in der älteren Bevölkerung in Deutschland bis 2020 und Konsequenzen für das Gesundheitssystem

Am Beispiel der Krebserkrankungen lassen sich die Probleme der demographischen Entwicklung und deren Auswirkungen auf das Gesundheitssystem gut abbilden. Nach Schätzungen des Robert Koch-Instituts [6] erkrankten 1998 in Deutschland insgesamt 206000 der über 65-Jährigen an Krebs, davon 99000 männlichen und 107000 weiblichen Geschlechts. Gegenüber 1990 bedeutet das einen Anstieg bei Männern um 15\% und bei Frauen um 2\%. Abbildung 2 zeigt, dass die - insbesondere bei Männern starken Zuwächse in den absoluten Erkrankungszahlen ausschließlich durch Altersstrukturveränderungen bedingt sind. Eine Altersstandardisierung auf die (alte) Europabevölkerung ergibt bei Männern wie bei Frauen über 65 Jahren eine relativ konstante Inzidenz. Dies rechtfertigt die vereinfachende Annahme einer auch künftig in der Größen- ordnung von 1998 zu erwartenden Krebsinzidenz für die Abschätzung zu erwartender Krebserkrankungszahlen.

Wie nachfolgend in Abb. 3 dargestellt, steigt die absolute Anzahl der Krebsneuerkrankungen bei über 65jährigen Männern bis zum Jahre 2020 auf insgesamt 157000 an. Dies entspricht gegenüber 1998 einem Anstieg um $60 \%$. Bei den Frauen steigt die Anzahl der Krebsneuerkrankten bis zum Jahre 2020 auf 132000 , der prozentuale Anstieg fällt mit $24 \%$ jedoch erheblich geringer aus als bei den Männern. Schon spätestens ab dem Jahr 2005 würden demnach im Altersbereich ab 65 Jahre absolut mehr Männer als Frauen an Krebs erkranken.

Auch wenn die Annahme eines im Laufe der Jahre konstant gebliebenen Krebserkrankungsrisikos stark vereinfachend ist, können selbst besser angepasste Modelle nichts an der Grundaussage ändern, dass die absolute Zahl der Krebspatienten und damit die Beanspruchung des Gesundheitswesens deutlich und kontinuierlich zunehmen wird.
„DieabsoluteZahl der Krebspatienten und damit die Beanspruchung des $\mathrm{Ge}$ sundheitswesens wird in den kommenden zwanzig Jahren deutlich und kontinuierlich zunehmen."

Dies hat schon bei gleichbleibenden durchschnittlichen Kosten für die Grundversorgung Krebskranker eine Vervielfachung der Kosten zur Folge. Berücksichtigt man aber darüber hinaus den Forschungsbedarf auf dem Gebiet der Krebstherapie, mit dem Ziel wirkungsvolle Behandlungsmethoden zu entwickeln, so erhöhen sich die Kosten weiter. Folgt man den Prognosen von Strohmeyer und Weißbach [7], so werden sich die Ausgaben speziell für onkologische Arzneimittel in den nächsten fünf Jahren weltweit nahezu verdoppeln. Andere nicht minder kostspielige Therapieformen werden ebenfalls entwickelt. Diese Kostenexplosion, die weit über den durch die wachsenden Patientenzahlen zu erwartenden Anstieg hinausgeht, bedarf wohl einer intelligenteren Strategie als einer formalen „Deckelung" von Budgets oder der Anhebung von Versicherungssätzen.

Tabelle 3

Zwölf-Monatsprävalenzen chronischer Krankheiten (in \%) nach Altersgruppen

\begin{tabular}{|c|c|c|c|c|c|c|}
\hline \multirow[t]{2}{*}{ Auswahl } & \multicolumn{6}{|c|}{ Altersgruppen (in Jahren) } \\
\hline & 20 bis 29 & 30 bis 39 & 40 bis 49 & 50 bis 59 & 60 bis 69 & 70 bis 79 \\
\hline Hypertonie & 1,8 & 4,8 & 10,4 & 24,9 & 39,3 & 49,4 \\
\hline Durchblutungsstörungen am Herzen & 0,1 & 0,3 & 0,3 & 4,3 & 11,9 & 19,1 \\
\hline Herzschwäche, Herzinsuffizienz & 0,0 & 0,2 & 0,8 & 2,0 & 5,8 & 16,3 \\
\hline Schlaganfall & 0,1 & 0,0 & 0,1 & 0,3 & 0,8 & 1,5 \\
\hline Durchblutungsstörungen am Gehirn & 0,0 & 0,2 & 0,3 & 1,2 & 2,4 & 4,0 \\
\hline Durchblutungsstörungen der Beine & 0,0 & 0,5 & 0,7 & 2,6 & 6,0 & 10,3 \\
\hline Diabetes mit Insulin & 0,2 & 0,4 & 0,8 & 1,2 & 2,3 & 4,7 \\
\hline Diabetes ohne Insulin & 0,1 & 0,2 & 1,0 & 3,6 & 8,4 & 11,2 \\
\hline Erhöhte Blutfette & 2,4 & 5,3 & 13,3 & 21,7 & 29,7 & 24,0 \\
\hline Harnsäureerhöhung & 0,3 & 1,7 & 4,8 & 8,0 & 9,5 & 10,4 \\
\hline Arthrose & 6,2 & 12,3 & 26,3 & 40,4 & 53,2 & 51,1 \\
\hline Polyarthritis & 1,6 & 1,7 & 3,5 & 6,7 & 6,0 & 7,7 \\
\hline Osteoporose & 0,0 & 0,2 & 1,2 & 3,9 & 8,6 & 11,9 \\
\hline Migräne & 8,6 & 11,2 & 12,8 & 12,4 & 4,8 & 3,6 \\
\hline Heuschnupfen & 20,0 & 16,7 & 11,8 & 9,9 & 7,2 & 4,5 \\
\hline Kontaktekzem & 8,6 & 9,4 & 7,4 & 6,4 & 4,5 & 2,8 \\
\hline Neurodermitis & 4,9 & 2,9 & 2,2 & 1,6 & 1,1 & 1,7 \\
\hline Nahrungsmittelallergie & 3,4 & 4,9 & 3,7 & 2,1 & 1,5 & 1,1 \\
\hline $\begin{array}{l}\text { Durchschnittliche Anzahl von Krankheiten } \\
\text { je } 100 \text { Personen in der Altersgruppe }\end{array}$ & 103,9 & 124,1 & 166,0 & 240,0 & 302,6 & 336,1 \\
\hline
\end{tabular}

Quelle: Bundes-Gesundheitssurvey 1998. 


\section{Originalien und Übersichtsarbeiten}

Tabelle 4

Anzahl von Krankheiten (in \%); 65 bis 70 Jahre

\begin{tabular}{|c|c|c|c|c|c|c|c|c|}
\hline & \multicolumn{4}{|l|}{ Ost } & \multicolumn{4}{|l|}{ West } \\
\hline & \multicolumn{2}{|l|}{ 1990/91 } & \multicolumn{2}{|l|}{1998} & \multicolumn{2}{|l|}{$1990 / 91$} & \multicolumn{2}{|l|}{1998} \\
\hline & Männer & Frauen & Männer & Frauen & Männer & Frauen & Männer & Frauen \\
\hline Keine & 3,7 & 0,9 & 5,1 & 9,2 & 0,6 & 0,9 & 5,7 & 6,0 \\
\hline Eine & 10,7 & 7,3 & 3,8 & 5,0 & 6,9 & 5,8 & 6,4 & 3,5 \\
\hline Zwei u. mehr & 85,7 & 91,8 & 91,0 & 85,9 & 92,6 & 93,3 & 88,0 & 90,6 \\
\hline
\end{tabular}

Tabelle 5

Zufriedenheit mit der Gesundheit (in \%); 65 bis 70 Jahre

\begin{tabular}{|c|c|c|c|c|c|c|c|c|}
\hline & \multicolumn{4}{|l|}{ Ost } & \multicolumn{4}{|l|}{ West } \\
\hline & \multicolumn{2}{|l|}{ 1990/91 } & \multicolumn{2}{|l|}{1998} & \multicolumn{2}{|l|}{ 1990/91 } & \multicolumn{2}{|l|}{1998} \\
\hline & Männer & Frauen & Männer & Frauen & Männer & Frauen & Männer & Frauen \\
\hline Sehrunzufrieden & 18,4 & 16,2 & 9,9 & 9,6 & 18,1 & 13,6 & 12,8 & 6,3 \\
\hline Mittelm.zufrieden & 59,6 & 36,9 & 53,2 & 49,2 & 52,4 & 46,5 & 46,5 & 45,1 \\
\hline Sehr zufrieden & 22,0 & 46,8 & 36,8 & 41,2 & 29,5 & 39,9 & 40,6 & 48,6 \\
\hline
\end{tabular}

Tabelle 6

Zufriedenheit mit dem Leben (in \%); 65 bis 70 Jahre

\begin{tabular}{|c|c|c|c|c|c|c|c|c|}
\hline & \multicolumn{4}{|l|}{ Ost } & \multicolumn{4}{|l|}{ West } \\
\hline & \multicolumn{2}{|l|}{$1990 / 91$} & \multicolumn{2}{|l|}{1998} & \multicolumn{2}{|l|}{$1990 / 91$} & \multicolumn{2}{|l|}{1998} \\
\hline & Männer & Frauen & Männer & Frauen & Männer & Frauen & Männer & Frauen \\
\hline Sehr unzufrieden & 0,0 & 2,0 & 2,8 & 0,0 & 2,9 & 1,4 & 0,9 & 0,6 \\
\hline Mittelm. zufrieden & 62,0 & 40,0 & 32,5 & 31,6 & 49,1 & 44,1 & 26,1 & 22,4 \\
\hline Sehr zufrieden & 38,0 & 58,0 & 64,7 & 68,4 & 48,0 & 54,5 & 73,0 & 77,0 \\
\hline
\end{tabular}

Der tendenzielle Grenznutzen wachsender medizinisch-technischer Aufwendungen wird zunehmend erkennbar. Der Erhalt bzw. die weitere Verbesserung der gesundheitlichen Situation der Bevölkerung bedarf immer größerer Aufwendungen an materiellen, finanziellen und personellen Ressourcen seitens der Gesellschaft. Die zunehmende soziale Asymmetrie der durch die medizinische Versorgung verbrauchten finanziellen Mittel und Ressourcen und der Ergebnisse der medizinischen Versorgung ist bisher eine ungenügend beantwortete Herausforderung. Die Herbeiführung eines gesellschaftlichen Konsenses über finanzierbare Prioritäten im
Rahmen der Solidargemeinschaft bei gleichzeitiger Qualitätssicherung der medizinischen Versorgung (Entwicklung von Therapiestandards als Element der evidence based medicine) ist zukünftig unabdingbar. Gleichzeitig ist jedoch der Hinweis auf die Bedeutung von Prävention und Früherkennung [8] als wirksamste und kostengünstigste Methode zur Vermeidung von Krebserkrankung und Krebstod ein Schritt weg von der Anspruchsgesellschaft hin zur Wahrnehmung von eigener Verantwortung für die Gesundheit.

\section{Inanspruchnahme ambulanter Versorgung durch ältere Menschen}

Die Ergebnisse des Bundes-Gesundheitssurveys belegen, dass mit zunehmendem Lebensalter die Zahl der Arztkontakte pro Jahr zunimmt [9]. Dabei hängt die Zahl der Arztkontakte in allen Altersgruppen stark mit der subjektiven Einschätzung der eigenen Gesundheit zusammen (siehe Abb.4). Interessanterweise zeigt ein Vergleich der in der Erhebung 1998 gefundenen Arztkontaktrate mit der 1991/92 gefundenen, dass fast durchgängig sowohl bei Frauen als auch bei Männern in Ost wie in West die 65bis 70-Jährigen 1998 häufiger im letzten 
Vierteljahr beim Arzt waren als noch 1991 (Tabelle 7).

Dies geht einher mit der zuvor erwähnten wachsenden Zufriedenheit mit der eigenen Gesundheit und lässt Raum für allerlei Mutmaßungen zur Begründung. Ein Ansatz zur Erklärung wäre der, dass die Inanspruchnahme der medizinischen Versorgung zunimmt, weil die Versorgungsinhalte zunehmend auf Linderung, funktionelle Kompensation, aufschiebende Wirkung von Krankheitsepisoden und Aufrechterhaltung der Selbständigkeit gerichtet sind.

\section{Arzneimittelkonsum älterer Menschen}

Der Arzneimittelkonsum steigt mit zunehmendem Alter an. Dies belegen die Zahlen des im Rahmen des Bundes-Gesundheitssurveys durchgeführten Arzneimittelsurveys $[10,11]$. Dabei wächst sowohl der Prozentsatz der Arzneimittelanwender als auch die durchschnittlich angewandte Zahl der Arzneimittel. Der altersspezifische Anstieg beginnt für den überwiegenden Teil der Arzneimittelgruppen bereits in den Altersgruppen 40 bis 50 bzw. 50 bis 60 Jahre. Der in Tabelle 8 dargestellte Anstieg im Alter von 65 bis 80 Jahren fällt dagegen vergleichsweise niedrig aus.

Die durchschnittliche Zahl der angewandten Arzneimittel ist ebenfalls altersabhängig und liegt bei Frauen deutlich über den bei den Männern registrierten Zahlen (siehe Tabelle 9).

Die Arzneimittelgruppen, (7-TageBefragungsfenster), bei denen eine besonders starke Steigerung der Anwendungshäufigkeit im Alter zwischen 65 und 80 Jahren beobachtet werden kann, sind

\begin{tabular}{|c|c|}
\hline Aoo & $\begin{array}{l}\text { Alimentäres System } \\
\text { und Stoffwechsel, }\end{array}$ \\
\hline $\mathrm{Coo}$ & Kardiovaskuläres System, \\
\hline Goo & $\begin{array}{l}\text { Urogenitalsystem } \\
\text { (bei Männern), }\end{array}$ \\
\hline Moo & $\begin{array}{l}\text { Muskel- und Skelettsystem } \\
\text { (bei Frauen), }\end{array}$ \\
\hline Noo & Nervensystem. \\
\hline
\end{tabular}

Bei den anderen Arzneimittelgruppen stagniert die Arzneimittelanwendung im Wesentlichen oder weist sogar rückläufige Tendenzen auf. Für einige Arzneimittel kann festgestellt werden, dass

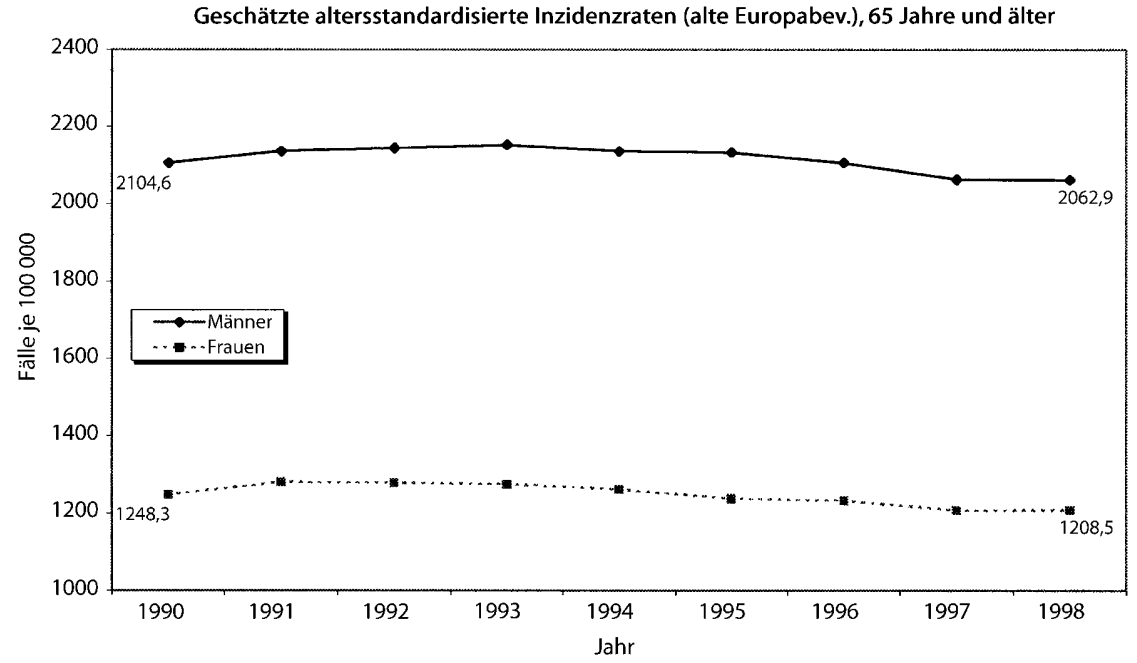

Abb.2 $\triangle$ Entwicklung der Krebsinzidenz in Deutschland in der älteren Bevölkerung

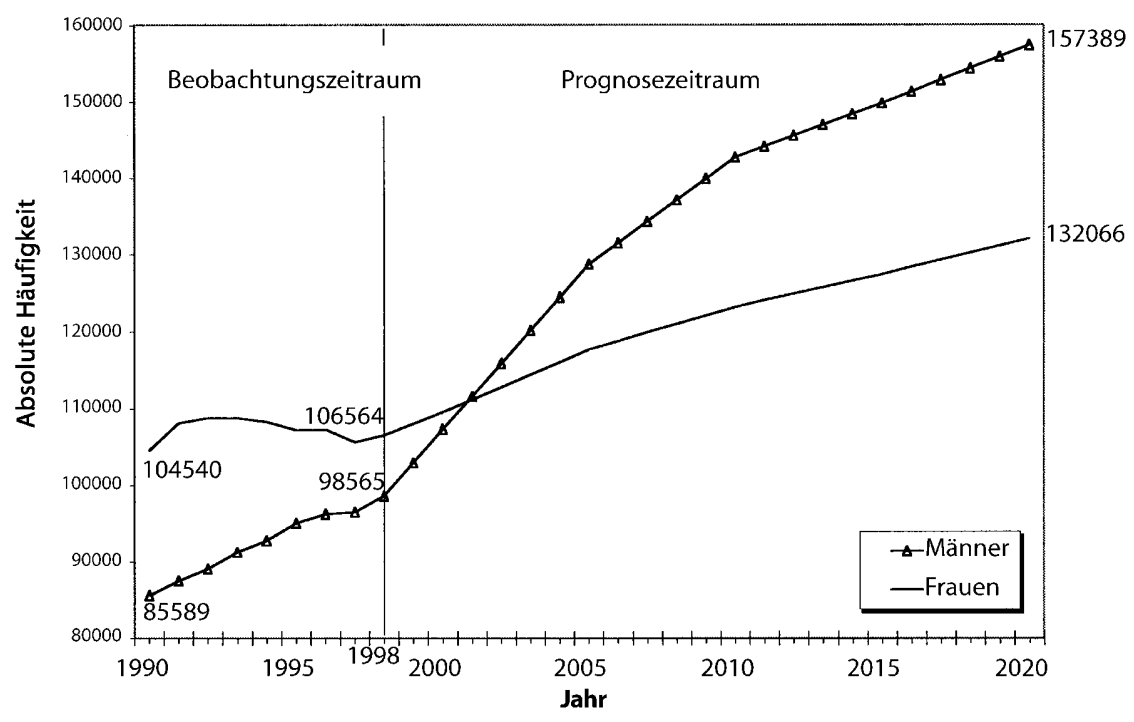

Abb.3 $\Delta$ Geschätzte Zahl jährlich neu an Krebs Erkrankender in Deutschland, 65 Jahre und älter (konst. Erkrankungsrisiko von 1998)

sich in der Altersgruppe der 70- bis 74Jährigen ein Gipfel der Anwendungshäufigkeit zeigt, der mit weiter steigendem Alter wieder etwas abnimmt.

Die in den alten Bundesländern seit 1985 bereits wiederholt durchgeführten Arzneimittelsurveys lassen erkennen, dass die durchschnittliche Zahl der angewendeten Medikamente in der Gesamtbevölkerung kontinuierlich steigt. Für die Gruppe der 65- bis 70-Jährigen hat sich beispielsweise die Zahl von 2,5 im Jahr 1985 auf 3,14 im Jahr 1998 (Männer) bzw. für Frauen von 3,34 auf 4,22 erhöht.

\section{Demographischer Wandel und Gesundheit: Datenlage und Schlussfolgerungen}

Die vorangegangenen Ausführungen belegen, dass es zwar partiell Daten gibt, die darauf hindeuten, dass die Inanspruchnahme medizinischer Versorgung zunehmen wird, dass der Alterungsprozess unserer Bevölkerung aber nicht zu einem proportionalen Anstieg der Krankheits- und Behinderungslast für das gesundheitliche Versorgungssystem führen wird. Um allerdings ähnlich umfassende Prognosen für gesundheitliche Entwicklungen zu geben, wie sie das Statistische Bundesamt für die zu er- 


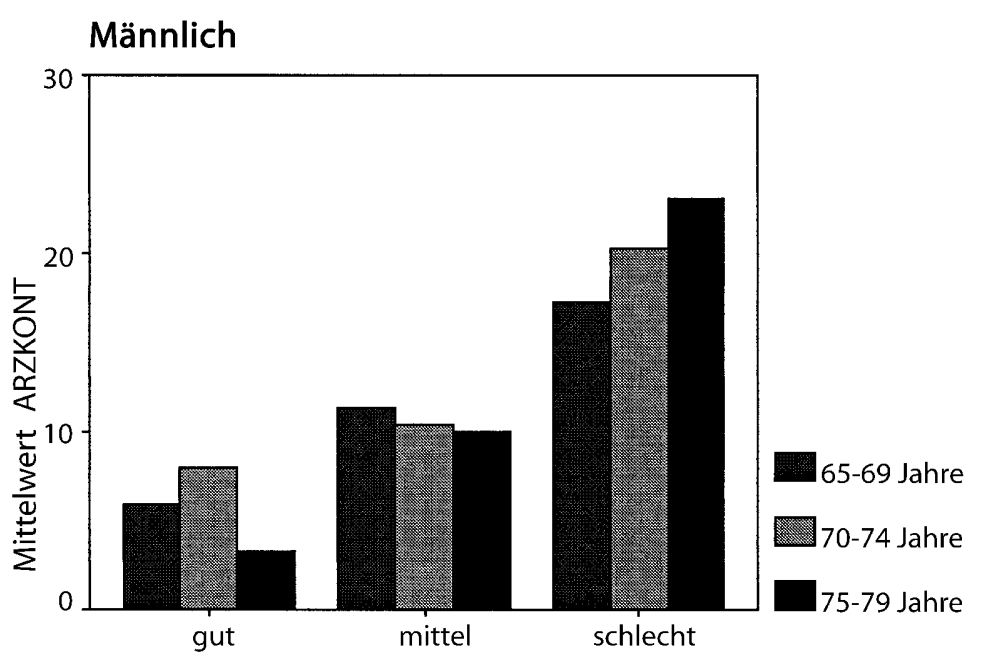

Einschätzung des Gesundheitszust.

Fälle gewichtet nach W98

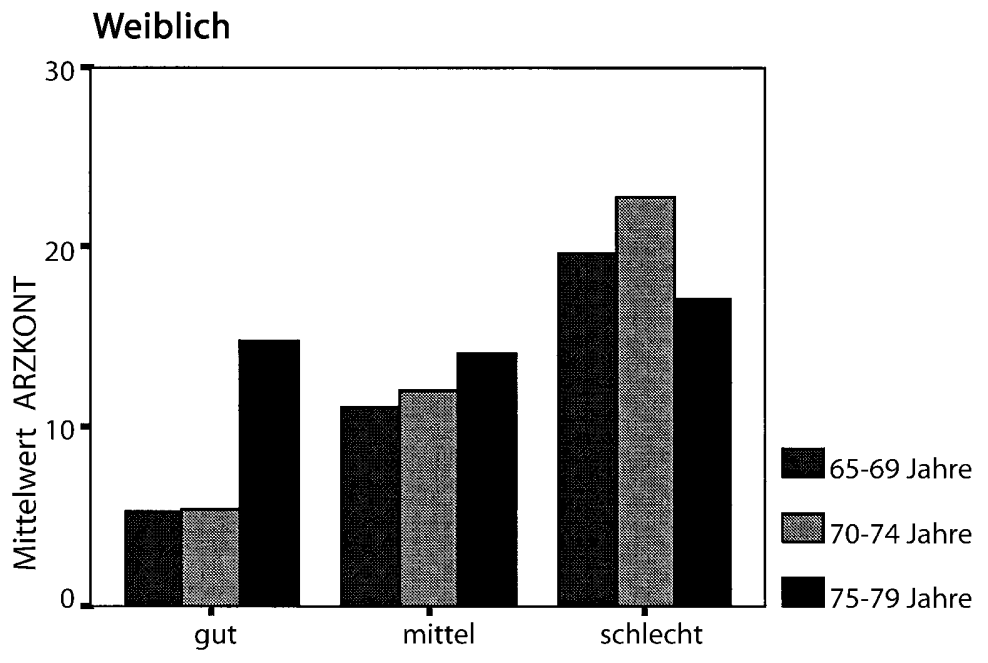

Einschätzung des Gesundheitszust.

Fälle gewichtet nach W98

Abb. $4 \Delta$ Einschätzung des Gesundheitszustandes (Fälle gewichtet nach W98) wartende demographische Entwicklung gegeben hat, reichen die vorhandenen Daten nicht aus. Doch selbst solche Prognosen wie die der künftigen Lebenserwartung (einer Kennzahl, die auf sehr umfangreichem und kontinuierlich erhobenem Datenmaterial beruht) sind in ihrer Güte erst dann richtig zu bewerten, wenn der prognostizierte Zeitpunkt eingetreten ist.

\section{„Der Alterungsprozess der Bevölkerung wird nicht zu einem proportionalen Anstieg der Krankheits- und Behinderungs- last für das gesundheitliche Versorgungssystem führen."}

So wurde im Jahr 1971 in einem vom Bundesminister für Jugend, Familie und Gesundheit herausgegebenen Gesundheitsbericht [12] ebenfalls eine Prognose der künftigen Lebenserwartung gegeben: 1971 war ein Anstieg der durchschnittlichen Lebenserwartung von damals ca. 70 Jahren auf 85 Jahre im Jahr 1990 prognostiziert worden, tatsächlich stieg die Lebenserwartung aber nur auf ca. 77 Jahre. Die Zuwachsprognose für diese Kennzahl, die einen Kardinaleinfluss auf den Bedarf an medizinischer Versorgung hat, war also um mehr als das Doppelte zu hoch.

Wie störanfällig relativ stabil erscheinende Trends in der Mortalität gegenüber äußeren Einflüssen sind, zeigen die in Tabelle 10 dargestellten Zahlen zur vorzeitigen Sterblichkeit (Tod vor Vollendung des 65. Lebensjahres). In der unmittelbaren Transformationsphase des gesellschaftlichen Systems in den neuen Bundesländern (1990 bis 1993) ist insbe-

Tabelle 7

Inanspruchnahme (letzter Arztkontakt), 65 bis 70 Jahre (in \%)

\begin{tabular}{|c|c|c|c|c|c|c|c|c|}
\hline \multirow{3}{*}{$\begin{array}{l}\text { Letzter } \\
\text { Arztkontakt } \\
\text { liegt zurück: }\end{array}$} & \multicolumn{4}{|l|}{ Ost } & \multicolumn{4}{|l|}{ West } \\
\hline & \multicolumn{2}{|l|}{$1990 / 91$} & \multicolumn{2}{|l|}{1998} & \multicolumn{2}{|l|}{$1990 / 91$} & \multicolumn{2}{|l|}{1998} \\
\hline & Männer & Frauen & Männer & Frauen & Männer & Frauen & Männer & Frauen \\
\hline Max. 4 Wochen & 76,5 & 75,1 & 70,5 & 66,8 & 66,2 & 60,0 & 59,8 & 64,5 \\
\hline 2- 3 Monate & 3,1 & 9,5 & 15,3 & 22,0 & 18,9 & 23,0 & 15,8 & 22,6 \\
\hline 4- 12 Monate & 12,3 & 8,5 & 6,1 & 4,9 & 10,8 & 13,9 & 15,9 & 8,4 \\
\hline Mehr als ein Jahr & 8,2 & 6,9 & 8,1 & 6,3 & 4,9 & 3,1 & 8,5 & 4,5 \\
\hline
\end{tabular}


Tabelle 8

Arzneimittelanwender ${ }^{2}$

\begin{tabular}{lcc} 
& Männer & Frauen \\
\hline 65-69 Jahre & $85,9 \%$ & $93,4 \%$ \\
70- 74 Jahre & $86,7 \%$ & $91,5 \%$ \\
75- 79 Jahre & $88,0 \%$ & $93,9 \%$
\end{tabular}

Tabelle 9

Durchschnittliche Zahl der

angewendeten Medikamente ${ }^{3}$

\begin{tabular}{lll} 
Altersgruppe & Männer & Frauen \\
\hline 65-69 Jahre & 3,21 & 4,10 \\
70-74 Jahre & 3,64 & 3,99 \\
75- 79 Jahre & 3,92 & 4,73
\end{tabular}

sondere für Männer entgegen dem auch in Ostdeutschland generell vorliegenden Trend der Verringerung der Frühsterblichkeit eine Steigerung eingetreten, die einen „Rückschritt“ von ca. zehn Jahren darstellt. Die Tatsache, dass dieses Defizit innerhalb kürzester Zeit wieder aufgeholt und die Frühsterblichkeit seit 1995

\footnotetext{
2 Bundes-Gesundheitssurvey 1998 (gewichtete und vorläufige Zahlen)

3 Bundes-Gesundheitssurvey 1998 (gewichtete und vorläufige Zahlen)
}

jedes „Vorwendeniveau“ unterschreitet, beweist den Einfluss von zum Teil nicht vorhersehbaren sozialen Bedingungen auf gesundheitliche Entwicklungen.

Wie bereits mehrfach festgestellt, haben wir es hier mit komplexen Einflüssen zu tun, die in ihrer Wirkung teilweise gegenläufig, teilweise rekursiv sind. Hinzu kommt der Einfluss von Entwicklungen der Forschung, die von ihrer Natur her nicht prognostizierbar sind. Dies soll keineswegs das Plädoyer gegen jede Art von Prognosen sein, sondern vielmehr der Appell, weniger prophetisch, dafür vielleicht aber mehr „seismographisch“ sein zu wollen. Aber selbst für die „seismographischen“ Reaktionen ist die Datenlage zu verbessern.

Die Kombination zweier Ansätze, die methodisch und inhaltlich eng verzahnt werden, kann dieses Datendefizit entscheidend verbessern:

Ein großer Datenfundus zur Gesundheit und Versorgung in Deutschland - die Gesundheits- und Sozialdaten der Krankenversicherungen und der Kassenärztlichen Vereinigungen - liegt weitgehend brach. Die Aufbereitung und Nutzbarmachung dieser Daten, beispielsweise in Form einer Versichertenstichprobe, wäre mit vergleichsweise geringem finanziellen Aufwand verbunden und könnte auf Bevölkerungsebene zeitnah Veränderungen im Krankheitsspektrum und im Versorgungsverhalten und -angebot aufzeigen. Die auf der Grundlage von Daten Schweizer Krankenkas- sen im Jahr 1996 vorgelegte Studie zum Thema „Demographische Alterung und Gesundheitskosten: Eine Fehlinterpretation" [13] zeigt überzeugend, dass individuelle Risikofaktoren wie Übergewichtigkeit Kostenfaktoren sind, die das Alter als Einflussfaktor außer Kraft setzen. Die Schlussfolgerungen in Richtung Prävention und individuelle Eigenverantwortung liegen auf der Hand. Entsprechende Auswertungen wären prinzipiell auch für Deutschland möglich. Die konzeptionellen Grundlagen für die Erschließung dieser Daten als epidemiologische Datenquelle für die Gesundheitsforschung wurden im Rahmen der Gesundheitsberichterstattung des Bundes entwickelt [14].

Während die Daten der Krankenversicherung, vereinfacht gesprochen, die Interaktion von Gesundheit und Versorgung spiegeln, benötigen wir mehr Informationen, wie sich die Gesundheit und ihre Determinanten in Deutschland entwickeln, noch bevor es zu einem $\mathrm{Pa}$ tientenkontakt mit dem Gesundheitssystem kommt. Die Hauptdatenquelle für diesen Bereich sind bislang die mehrfach erwähnten Gesundheitssurveys. Diese Surveys erfolgen idealerweise häufig und mit einem Stichprobenumfang, der auch regionale Entwicklungen innerhalb Deutschlands zuverlässig erfasst. Je mehr Erhebungszeitpunkte zur Verfügung stehen, desto belastbarer werden sowohl Trendaussagen als auch die prognostischen Fenster.

\section{Tabelle 10}

Entwicklung der vorzeitigen Sterblichkeit ${ }^{1}$ (Gestorbene insgesamt 1 bis unter $65 \mathrm{~J}$ ahre) für alle Todesursachen (ICD 9: 001- 999) je 100000 der Bevölkerung; Bundesrepublik Deutschland (BRD); früheres Bundesgebiet/alte Bundesländer $(\mathrm{ABL})^{3)}$ und ehemalige DDR/neue Bundesländer (NBL)3),1986- 1997

\begin{tabular}{|c|c|c|c|c|c|c|c|c|c|c|c|c|}
\hline 1986 & 1987 & 1988 & 1989 & $1990^{21}$ & $1991^{2)}$ & $1992^{21}$ & $1993^{2)}$ & 1994 & 1995 & 1996 & 1997 & $\begin{array}{l}1986-1 \\
\text { in } \% 4)\end{array}$ \\
\hline \multicolumn{13}{|c|}{ he Bevölkerung } \\
\hline- & - & - & - & - & 378,5 & 367,5 & 362,0 & 353,0 & 342,7 & 332,1 & 317,6 & \\
\hline 374,0 & 363,2 & 357,6 & 352,9 & 349,3 & 350,0 & 340,0 & 336,9 & 329,5 & 322,6 & 314,8 & 302,5 & $-19,1$ \\
\hline 451,8 & 448,6 & 459,2 & 448,4 & 497,5 & 496,9 & 482,7 & 467,3 & 451,3 & 426,7 & 404,7 & 380,5 & $-15,8$ \\
\hline \multicolumn{13}{|c|}{ le Bevölkerung } \\
\hline- & - & - & - & - & 177,2 & 170,5 & 169,4 & 164,2 & 160,8 & 157,2 & 151,2 & \\
\hline 178,3 & 173,8 & 171,5 & 169,5 & 167,7 & 166,5 & 161,6 & 163,2 & 158,6 & 156,9 & 153,8 & 149,4 & $-16,2$ \\
\hline 227,4 & 218,4 & 220,0 & 213,6 & 225,5 & 218,5 & 205,1 & 193,9 & 186,6 & 176,7 & 171,0 & 158,7 & $-30,2$ \\
\hline
\end{tabular}

${ }^{1)}$ Standardisiert auf die „alte Europastandardbevölkerung ${ }^{\prime}{ }^{2)}$ Unmittelbare Transformationsphase in den neuen Bundesländern, ${ }^{3)}$ Bis 2. Oktober 1990 früheres Bundesgebiet und ehemalige DDR, ${ }^{4}$ Abnahme der Sterblichkeit von 1986 bis 1997 in Prozent. 
Die Kontinuität und Kombination dieser beiden Datenquellen liefern die Voraussetzungen für ein GesundheitsNavigationsinstrument. Die Informationen stellen die Grundlage von kurz- und langfristigen Entscheidungen und deren im Zeitverlauf erforderlich werdenden Modifikationen im Sinne einer evidence based policy.

Für ihre Zuarbeit und Hinweise zu diesem Text danke ich folgenden Mitarbeitern der Abteilung Epidemiologie und Gesundheitsberichterstattung des Robert Koch-Instituts:

Andreas Bergholz, Ute Ellert, Dr. Jürgen Grimm, Dr. Jörg Haberland, Dr. Hildtraud Knopf, Dr. Hans-Ulrich Melchert, Dr. Dieter Schön, Dr. Heribert Stolzenberg, Michael Thamm, Dr. Wolfgang Thefeld, Dr. Gerd Wiesner, Dr. Thomas Ziese.

\section{Literatur}

1. Statistisches Bundesamt, Wiesbaden (2000) Bevölkerungsentwicklung Deutschlands bis 2050. Ergebnisse der 9. Koordinierten Bevölkerungsvorausberechnung. Eigenverlag, Wiesbaden

2. Bellach BM, Knopf H,Thefeld W (1998) Der Bundes-Gesundheitssurvey 1997/98. Gesundheitswesen. Sonderheft 2: S59- 68

3. Thefeld W, Stolzenberg H, Bellach B-M (1999) Bundes-Gesundheitssurvey: Response, Zusammensetzung der Teilnehmer und Non-Responder-Analyse. Gesundheitswesen [Sonderheft 2]:S57-61

4. Dinkel RH (1999) Demographische Entwicklung und Gesundheitszustand. Eine empirische Kalkulation der Healthy Life Expectancy für die Bundesrepublik auf der Basis von Kohortendaten. In: Häfner H (Hrsg) Gesundheit - unser höchstes Gut? Springer, Berlin Heidelberg New York Tokyo, S61-83

5. Zweiter Zwischenbericht der Enquête-Kommission "Demographischer Wandel - Herausforderungen unserer älter werdenden Gesellschaft an den einzelnen und die Politik" (1998). Deutscher Bundestag, 13. Wahlperiode, Drucksache 13/11460

6. Arbeitsgemeinschaft Bevölkerungsbezogener Krebsregister in Deutschland (1999) Krebs in Deutschland - Häufigkeiten und Trends, 2. aktual. Ausgabe. Eigenverlag, Saarbrücken

7. Strohmeyer T,Weißbach L (1999) Arzneimittelentwicklung in der Onkologie. Status und Ausblick unter besonderer Berücksichtigung des Standorts Deutschland. Dtsch Med Wochenschr 124:231-235

8. Weißbach, L (2000) Die Wahrheit über den Krebs. Frankfurter Rundschau 10.1.2000:6

9. Bergmann E, Kamtsiuris P (1999) Inanspruchnahme medizinischer Leistungen. Gesundheitswesen [Sonderheft 2]:S138-144

10. Bertelsmann A, Knopf H, Melchert HU (1998) Der Bundes-Gesundheitssurvey als pharmakoepidemiologisches Instrument. Gesundheitswesen [Sonderheft 2]: 589- 94

11. Knopf H, Melchert HU (1999) Subjektive Angaben zur täglichen Anwendung ausgewählter Arzneimittelgruppen - Erste Ergebnisse des Bundes-Gesundheitssurveys 1998. Gesundheitswesen [Sonderheft 2]: S151-S157

12. Bundesminister für Jugend, Familie und Gesundheit, Bonn (1971) Gesundheitsbericht 1971. Kohlhammer, Bonn

13. Zweifel P,Felder S, Meier M (1996) Demographische Alterung und Gesundheitskosten: Eine Fehlinterpretation. In: Oberender P (Hrsg) Alter und Gesundheit. Gesundheitsökonomische Beiträge 26:29- 46

14. Versichertenstichprobe aus der gesetzlichen Krankenversicherung (1999) Gesundheitsberichterstattung des Bundes. Statistisches Bundesamt, Eigenverlag, Wiesbaden

\section{Bösel/Rothkopf-Ischebeck Praktikum des Infektionsschutzes}

\section{Seiten, 21 Abbildungen, 10 Tabellen, 13. Auflage 2000, ISBN 3-87344-114-4, DM 58,00}

Altbekannte Infektionskrankheiten sowie neue und neuartige Erreger erfreuen sich in vielen Teilen der Welt einer Renaissance. Im Zeitalter von AIDS, BSE und MKS ist wohl jedem im medizinischen Bereich Tätigen und auch vielen Laien klar geworden, dass Infektionskrankheiten auch im Zeitalter von Impfstoffen, Antibiotika und ersten antiviralen Medikamenten noch lange nicht besiegt sind.

Um so zeitgerechter erscheint jetzt die neue Auflage des schon in vielen medizinischen Bereichen altbekannten und bewährten „Praktikum des Infektionsschutzes." Die Autoren beschreiben in sehr kompetenter Weise kurz und griffig die Klinik, Epidemiologie, Diagnostik, Therapie und Prophylaxe von 123 Infektionskrankheiten. Abbildungen und Tabellen lockern den Text auf. Man kann sich schnell und kompetent informieren. Die Autoren gliedern den Text in drei Teile. Man kann sich über die Infektionsprophylaxe durch Schutzimpfungen informieren, pränatale und perinatale Infektionen bilden einen gesonderten Abschnitt und im dritten Teil werden die diversen Infektionskrankheiten einzeln behandelt. Als kleinen Bonus enthält das Buch auch Aufgabenbeschreibung und Anschriften der Nationalen Referenzzentren für gesundheitlich besonders bedeutsame Infektionskrankheiten sowie auch die Ansprechpartner in den Konsiliarlaboratorien für weitere ausgewählte bakterielle, virale, parasitäre und mykotische Infektionen. Referenzzentren und Konsiliarlaboratorien sind geeignet, weitergehende Auskünfte zu einzelnen Infektionskrankheiten zu erteilen.

Das Buch erscheint in einem handlichen Format, ist sehr gut zum schnellen Nachschlagen geeignet und damit außergewöhnlich wertvoll für alle, die sich mit Infektionskrankheiten zu beschäftigen haben. Diesem Buch kann man vorbehaltlos eine große Verbreitung wünschen.

B-M. Kurth (Berlin) 